\title{
Aportes en torno a la paternidad y el cuerpo reproductivo masculino a partir de los hallazgos de tres estudios sobre varones y cuidados en Chile y México
}

\author{
Contributions about fatherhood and male reproductive body \\ regarding the findings of three studies concerning men and care \\ in Chile and Mexico
}

Marcelo Robaldo Salinas ${ }^{1}$

\begin{abstract}
Resumen
Al nivel más general este artículo aborda la mutua determinación entre la paternidad y la masculinidad a partir de las vivencias contemporáneas de varones heterosexuales y no heterosexuales en distintas sociedades de América Latina. En el marco de esta reflexión se comentan los hallazgos de tres estudios acerca de varones y paternidad presentados durante el V Coloquio Internacional de Estudios sobre Varones y Masculinidades 2015, usando como ejes de reflexión los conceptos de cuerpo reproductivo y homoparentalidad.
\end{abstract}

Palabras clave: masculinidad - paternidad - cuerpo reproductivo - homoparentalidad.

\begin{abstract}
At a general level this paper explores the relationship between fatherhood and masculinity within the everyday experiences of both heterosexual and non-heterosexual men from different Latin American societies. In this context of exploration and using the reproductive body and homosexual kinship as conceptual pivots, observations are made regarding the findings of three studies concerning men and fatherhood presented during the Fifth Man and Masculinities Studies International Congress 2015.
\end{abstract}

Key words: masculinity - fatherhood - reproductive body - homosexual kinship.

Fecha de recepción: Octubre 2015

Fecha de aprobación: Noviembre 2015

Sociólogo Universidad de Chile. Diplomado en Epistemología Feminista, Universidad Autónoma de México, Doctor (c) en Estudios de Género, Universidad de Londres. E-mail: marcelo.robaldo@gmail.com 
"Los cuerpos que han vivido en las sombras ¿cómo emergen ante la luz más brillante? ... lo hacen gracias a la solidaridad entre individuos heroicos".

Judith Butler en conferencia "Cuerpos que aún importan", 16 de septiembre de 2015, Universidad Nacional Tres de Febrero, Argentina.

\section{PRESENTACIÓN}

Durante el verano de 2015 tuve la oportunidad de participar como moderador de la mesa temática Paternidades, Hombres y Cuidados celebrada en el marco de los tres días de actividad del V Coloquio Internacional de Estudios sobre Varones y Masculinidades, en Santiago de Chile. Como parte de una reflexión en torno a la paternidad y el cuerpo desde de las vivencias contemporáneas de varones heterosexuales y no heterosexuales en distintas sociedades de América Latina, el presente trabajo comenta los hallazgos de tres estudios presentados en dicha mesa. Los tres estudios corresponden a: Arreglos parentales de varones gays en Ciudad de México: Entre la paternidad negada y la transformación inadvertida del cuidado, de Oscar Laguna (México), donde se investigan las prácticas de hombres gays en torno a la crianza y el cuidado dentro de la adversidad de la cultura mexicana; Los hombres siempre adoptan: Narrativas masculinas sobre reproducción asistida y adopción, de Florencia Herrera (Chile), que estudia por primera vez los relatos de la paternidad entre varones infértiles en el contexto de la sociedad chilena; Vínculo padre-hijo: Un análisis desde el itinerario biográfico de varones jóvenes no heterosexuales, de Rodrigo Lara-Quinteros y María Francisca Avendaño (Chile), un estudio que junta dos investigaciones acerca de la percepción de varones jóvenes homosexuales respecto de la paternidad y sus propios padres.

\section{INTRODUCCIÓN}

Pierre Bourdieu (2000) ha señalado que el orden social funciona como "una inmensa máquina simbólica que tiende a ratificar la dominación masculina en la que se apoya". En efecto, la realidad social en tanto dominación masculina es un orden donde los significantes de lo masculino colocan a ciertos varones en una condición de supremacía sobre las mujeres y otros varones. Este orden se estructura sobre la base de categorías de opuestos que reproducen y naturalizan la lógica jerarquizante y heteronormativa de la división sexual.

Para Mary Luz Sandoval un elemento central de la tesis de Bourdieu concerniente a la dominación masculina es que invierte totalmente la relación entre lo cultural y lo natural para explicar la división entre los sexos como principio de divisiones consiguientes (Sandoval, 2002). Siguiendo esta tesis podemos entender que la cultura en tanto sistema de oposiciones simbólicas entre lo femenino y lo masculino funda la inequidad social y determina las consiguientes formas de inequidad que imponen el orden de clases sociales y las formas de dominación racistas.

La fuerza de la ideología del género radica en que, entre otras cosas, divide a los seres humanos estrictamente entre mujeres y hombres y lo hace a partir del cuerpo. Un aspecto fundamental a considerar además es que dicha ideología prescribe la reproducción como misión por antonomasia para todos y cada uno de los cuerpos.

Sandoval además destaca que la dominación masculina opera por medio de la violencia simbólica (que es insensible, invisible para los dominados) y es admitida tanto por el dominado como por el dominador, ejerciéndose mediante el sentimiento, reconocimiento y sobre todo del conocimiento (2002). Como señala Bourdieu: 
Cuando los dominados aplican a lo que les domina unos esquemas que son el producto de la dominación, o, en otras palabras, cuando sus pensamientos y sus percepciones están estructurados de acuerdo con las propias estructuras de la relación de dominación que se les ha impuesto, sus actos de conocimiento son, inevitablemente, unos actos de reconocimiento, de sumisión (Bourdieu, 2000).

Debido a que en virtud de la dominación masculina las estructuras cognitivas siguen obedeciendo a la eternización de las divisiones sexuales es necesario detenerse brevemente en la idea específica de que el conocimiento científico de las ciencias sociales se encuentra inserto dentro de la dominación masculina.

\section{UNA EPISTEMOLOGÍA CRÍTICA DE LA DOMINACIÓN MASCULINA}

Las preguntas que se han hecho las epistemólogas del género sobre el paradigma de las ciencias sociales tienen suma relevancia, vistas desde la noción que la ciencia es en general una construcción social con arreglo a determinadas condiciones históricas, políticas e incluso sexuales. Desde ahí se puede entender que una pregunta elemental de la epistemología feminista es si acaso podemos decir que el conocimiento está "generizado", es decir, si tienen género la ciencia y la investigación científica. Son varias las epistemólogas feministas que contestan sí a esta pregunta.

Donna Haraway describe al positivismo como una falsa objetividad, ya que su perspectiva se ha construido únicamente desde la experiencia de los varones. La crítica al androcentrismo del conocimiento científico que Haraway desarrolla en su texto Ciencia, ciborgs y mujeres. La reinvención de la naturaleza nos lleva a pensar que efectivamente la ciencia en tanto una actividad practicada predominantemente por hombres construye una realidad a partir del sesgo masculino.

Por otro lado, en su ensayo ¿Existe un Método feminista? Sandra Harding plantea que las teorías tradicionales en las ciencias sociales se han aplicado de tal manera que hacen difícil comprender la participación de las mujeres en la vida social. Más aún, aunque las actividades de quienes mayoritariamente hacen ciencia (los hombres) son específicas del género masculino, estas no son representaciones de "lo humano".

En efecto, la ciencia tradicional ha construido un conocimiento desde la experiencia de los hombres para proyectar dicha experiencia como valor universal. Pero es necesario precisar que los hombres que dan voz a la ciencia tradicional pertenecen a una clase o a una raza dominante y que además son en su inmensa mayoría heterosexuales.

Parte del desafío para el estudio de las masculinidades consiste en dar visibilidad a prácticas y experiencias que expresan construcciones de masculinidad marginalizadas por las epistemes dominantes. Esto es precisamente el mérito de los estudios de Herrera, Laguna, Avendaño y Lara-Quintero, pues como señala la misma Herrera en el marco conceptual de su investigación: los estudios que se enfocan en la percepción masculina sobre la reproducción son escasos y suelen estar basados en la mirada de las mujeres sobre los hombres(). Se han realizado aún menos estudios que den cuenta de la mirada de los hombres en países en vías de desarrollo.

Efectivamente, para la ciencia masculinista no tienen voz aquellos hombres marginalizados por su condición de raza, clase o sexualidad. Al rescatar estas voces perdidas haremos bien 
en recordar lo que señala Harding respecto de que un problema es siempre un problema para alguien (Harding, 1987). Precisamente las tres investigaciones aquí vistas logran problematizar distintas formas de paternidad no hegemónicas porque visibilizan a hombres para quienes ser padres o hijos no es lícito, ya sea en virtud de la condena moral que acarrea socialmente su opción sexual o de su "incapacidad" de engendrar. Más aún, como se he planteado con anterioridad (Robaldo, 2011), la investigación acerca de varones y paternidad en la región necesita traspasar el cerco de lo que podemos llamar una epistemología heteronormativa.

\section{CUERPOS QUE AÚN IMPORTAN}

Hablando recientemente en una conferencia en la periferia de Buenos Aires Judith Butler ha reiterado la idea que los cuerpos importan, que reflexionar de la manera en que nuestros cuerpos impactan en la organización de nuestra convivencia es una cuestión de la mayor relevancia. No hay duda que para la comprensión del orden de género es básico considerar la paradoja que surge entre el cuerpo tomado en su materialidad y el cuerpo entendido como construcción social, como discursos construidos social e históricamente que definen sus sentidos.

Butler señala que los discursos de la ciencia respecto del cuerpo producen una poderosa verdad; que el sexo se puede definir en virtud de la posición relativa que cada uno ocupa en la vida reproductiva. La idea que la reproducción del cuerpo sexuado se encuentra al centro del orden de género da una vuelta de tuerca a la definición tradicional de la que estamos acostumbrados.

Butler afirma que las funciones reproductivas del hombre otorgan sentido a sus diferentes atributos de género, mientras que las funciones reproductivas de las mujeres le dan sentido a la definición de mujer. Dicho esquema es obviamente poderoso en nuestra cultura y prueba de esto es que cuando existen cuerpos que no son reproductivos ya sea por opción o por no contar con esa "capacidad" estos se vuelven problemáticos. Este es el caso de los varones infértiles que entrevista Herrera en su investigación.

\section{EL CUERPO REPRODUCTIVO}

Para pensar la infertilidad Florencia Herrera se sitúa dentro del campo de las relaciones sociales, buscando ir más allá de las definiciones médicas que han dominado su estudio. En efecto, el cuerpo ha tenido durante siglos el sustento de su "verdad" científica en la medicina y biología, pero en el marco de la modernidad este discurso ha terminado por propiciar la transformación del sentido monolítico del cuerpo, de la concepción que el sexo biológico, el género y la reproducción forman un todo en el que estos elementos están naturalmente unidos.

La desvinculación entre aspectos que fueron tradicionalmente elementos constitutivos de esta verdad del cuerpo, como son la sexualidad y la reproducción, ha permitido la deconstrucción de los esencialismos biologicistas presentes en los discursos sobre lo masculino y lo femenino de quienes incluso han reivindicado la igualdad de género. Como señala Mary Luz Sandoval,

fueron las ciencias de la naturaleza, paradójicamente, como la fisiología, la biología y luego la genética, las que sin pretenderlo, quebrantaron el sistema de explicación biológica de la supuesta inferioridad femenina, gracias a los descubrimientos sucesivos del óvulo en las hembras, de los cromosomas sexuales y su papel en la determinación del sexo del niño, del descubrimiento de los períodos fecundos e infecundos, lo que obligó a reconocer que "la naturaleza había programado el placer sexual de la mujer independientemente de la finalidad de la reproducción.". Asífue como comenzó su desalienación respecto de la naturaleza. Y fue 
también el punto de partida para deslindar campos que se confundían entre sexualidad, reproducción, maternidad y educación. De esa forma, aparecían con más claridad los aspectos culturales: teologías, ideologías, o aspectos socioeconómicos, estructuras de poder, división de roles, como finalidades naturales enmascaradas y abusivamente empleadas para fundar sistemas de representación aseguradores de la dominación masculina.

Este deslindar de la sexualidad, la reproducción, la maternidad y también la paternidad nos permite avanzar en la desconstrucción del cuerpo reproductivo. Como señala Butler Si la reproducción es el único método para pensar el cuerpo sexuado, dejamos fuera muchos aspectos, lo limitamos, agregando, para referirse al cambio de género, que pasar de $\mathrm{M}$ a F, o de F a M no implica necesariamente permanecer dentro del marco binario del género, es más bien convertir a la transformación en si el significado del género... el cambiarse es el vehículo del género en sí.

Según Butler hay que ir más allá de la descripción positivista de la materialidad del cuerpo y pensarlo en tanto campo de relaciones sociales, para lo que se hace necesario expandir el paradigma de la sexualidad más allá de las fronteras puestas por las definiciones determinadas por la reproducción sobre el cuerpo sexuado.

Los hallazgos del estudio de Herrera muestran cómo en la construcción del cuerpo reproductivo de sus entrevistados se cruzan la fertilidad y la virilidad, pues para estos varones lo natural es poder tener hijos, es decir, ser fértiles. Esta construcción del cuerpo masculino como un cuerpo reproductivo implica para ellos, hombres infértiles, un sufrimiento que no pueden expresar, pues consideran que su papel debe ser el de apoyar a sus parejas, quienes ellos consideran que son los que realmente sufren con los tratamientos médicos de la inseminación artificial o in vitro.

\section{LA PATERNIDAD EMERGENTE}

Los estudios acerca de masculinidad y paternidad en Chile a fines de los noventa y comienzos de la década del 2000 (Alméras, 1997; Valdés y Olavarría, 1998) revelan que los varones invierten mucho menos tiempo que sus parejas mujeres tanto en el cuidado de los hijos como en las labores domésticas. Esta situación se mantiene más o menos constante hasta el presente a nivel global, como lo revela el Informe Global sobre Paternidad de 2015.

Resultados similares muestra la investigación concerniente al tema en Argentina (Wainerman, 2007; Cosse, 2009), que señalan que aun cuando ya en los años setenta existía una mayor participación de los varones en la crianza y en el espacio doméstico esto no se traducía en igualdad de condiciones para ambos géneros.

Huelga preguntarse si estos hallazgos son evidencia de un estancamiento en los procesos de cambio en las relaciones de género -tal como señala Hochschild (1989) al hablar de una revolución estancada para referirse al aumento de las mujeres en doble jornada no acompañada por un aumento en la participación de los hombres en la esfera doméstica- o si se trata más bien de un estancamiento en la manera que la investigación se ha preguntado por la paternidad.

En este sentido pocos autores describen mejor el desarrollo de un modelo emergente de paternidad en América Latina que Isabella Cosse (2009), quien en su artículo La emergencia de un nuevo modelo de paternidad en Argentina revisa el desarrollo de dicho modelo en el transcurso de tres décadas, entre los años 50 y 70. Mediante la revisión de material que 
refleja las pautas culturales de la sociedad argentina, como revistas, películas, libros, columnas periodísticas y series de televisión, la autora da cuenta de la transformación del modelo de paternidad desde un padre autoritario, distante y proveedor exclusivo hacia una figura cercana, afectiva e involucrada en la crianza.

Nos detendremos en el caso argentino para apreciar los paralelos y contrastes entre alguno de sus aspectos y los hallazgos de las investigaciones que estamos comentando.

Cosse señala que durante los años 50 la ciencia médica se hace parte del discurso que norma cuáles debían ser los parámetros dentro de los que ha de desempeñarse la paternidad. Se estimaba que esta, la paternidad, no podía tener un carácter instintivo sino que debía ser el producto de la sociedad y la cultura. Para el modelo, que en esta etapa se difundió entre un segmento social reducido que se suponía culto y de alto poder adquisitivo, la autoridad del padre debía ser el resultado natural de la confianza, el cariño y el respeto. Es interesante ver cómo entre los jóvenes homosexuales entrevistados por Lara-Quinteros esta noción del padre en tanto autoridad no tiene mayor legitimidad, ya que, como señala el autor, en general ellos no otorgan un reconocimiento o una validación efectiva a este patrón normativo en tanto es percibido como algo lejano y poco alcanzable. Esto se explica porque la figura del padre para estos entrevistados en primera instancia aparece como lejana y silenciosa. En este sentido, el padre se vislumbra como un sujeto débil, cuya ausencia se da en términos no solo reales sino que también simbólicos.

Volviendo al estudio de Cosse, ella señala que en este periodo existe una convicción que la paternidad brotaría espontáneamente de una toma de conciencia respecto de lo que implicaba ser padre. ${ }^{2}$ Para el modelo de paternidad era fundamental mantener una diferenciación entre los progenitores, por lo que se planteaba dentro de una distribución complementaria de roles de género. Podemos apreciar que cómo se mantenía a pie firme la norma heterosexual de familia y parentesco.

Lo anterior tiene su paralelo en la construcción de familia hecha por los hombres estudiados por Herrera. Sus narraciones trasuntan un orden de género de opuestos complementarios en que él es vigoroso y protector y ella es sacrificada y dependiente y muestran cómo un segmento de la sociedad chilena está anclada en este modelo de familia. Similarmente, los entrevistados de Avendaño declaran que las ganas de concretar la paternidad surgirían cuando se tiene pareja y comienzan a crearse planes de familia. Como indica la misma Avendaño esto evidenciaría que el proyecto de vida de estos varones se relaciona con prácticas heteronormadas, las que son inculcadas durante todo el desarrollo de la persona, tanto en el contexto familiar como en el educacional, recalcando roles de género y secuencias determinadas para el curso de la vida.

Cosse señala que durante los sesenta se instala la perspectiva psicológica de la infancia que implica dejar de lado la evaluación moral de las conductas infantiles, para evaluarlas según criterios de normalidad o patología en el contexto del desarrollo psicológico. En ese marco se señala que la finalidad de una buena crianza era la estabilidad psicológica de los niños, para lo cual cobraron renovada importancia la autonomía y el rechazo a la violencia física. Durante estos años el modelo de la nueva paternidad adquirió creciente difusión en amplios segmentos del público. Para la tranquilidad de los padres se insistía en que las nuevas pautas

Esta noción de la paternidad, que implica cierto voluntarismo acerca del desarrollo de una paternidad alternativa a partir de una toma de conciencia de los hombres, sigue presente en las campañas que promueven una paternidad participativa tanto en Chile como internacionalmente. 
no herían la virilidad, ni significaban que los padres reemplazasen a las madres en las tareas consideradas naturalmente femeninas.

En esta vena de particular popularidad lograron los libros y talleres educativos de una asistente social, Eva Giberti, quien desde una noción psicoanalítica tradicional de los roles de género, dice Cosse, insistía en que la autoridad paterna ya no debía basarse en la imposición y la fuerza, sino en el diálogo y la comprensión, en forma concordante con las transformaciones modernas de la familia, en las cuales el pater podía compartir con la mujer el trabajo fuera del hogar y las responsabilidades políticas en la sociedad, pero esto no significaba que la figura del padre pudiese ser transferible (Cosse, 2009).

Señala Cosse que a medida que se complicó el concepto de paternidad se identificaron ciertos riegos que conllevaba el ejercicio de la paternidad, el padre debía mantener el equilibrio en su desempeño entre las distintas demandas que implica su papel y no permitir la aparición de "deviaciones" (el término viene de la propia literatura de la época) en la formación del rol sexual de la prole, siendo las más inquietantes la homosexualidad y la delincuencia de los hijos varones. En general existía la idea entre diversos autores locales como estadounidenses y europeos, Pichón Rivière entre otros, que la debilidad del padre en la formación de los hijos podía llevar a conductas desviadas.

Durante los setenta el modelo de paternidad por primera vez promueve que el padre traspase la división de roles de género de la domesticidad. Las revistas especializadas de esos años promovían que el padre tuviese la misma implicación en relación con los hijos que la madre. La importancia de la figura paterna ya no se apoyaba en las consecuencias peligrosas de su ausencia, sino en las influencias positivas de la paternidad activa y en las gratificaciones de la tarea en sí misma. Cosse escribe:

En los años setenta la nueva paternidad se había instalado como un paradigma preciso y potente que enfatizaba la importancia de la compenetración afectiva de los padres en las actividades de los niños (juegos, escuela y paseos), en el ejercicio de una autoridad basada en el diálogo y el respeto que contuviesen y pusiesen límites a los hijos, pero que también suponía que los progenitores se ocupasen de tareas que solían considerarse femeninas, generando así una ruptura al orden de género instituido.

En esta década la nueva paternidad ganó más terreno en el orden de los mandatos que en el de las prácticas. Sin embargo, más allá del problema de la incorporación a las prácticas cotidianas, las actitudes paternas del nuevo modelo comenzaron a quedar integradas en las auto-representaciones de ciertos padres.

Podemos encontrar algo de este aspecto del modelo en los hallazgos de Avendaño quien señala que para algunos entrevistados la propia familia -que presumiblemente incluye a los padres- es la base para las potenciales labores de crianza, porque de ella se desprenden los comportamientos que involucrarán el ejercicio de la paternidad.

Más aún, resulta sumamente interesante destacar cómo uno de los entrevistados de Lara-Quinteros (recordemos que se trata de jóvenes homosexuales) dio cuenta de mantener una estrecha relación con su padre, marcada por la comunicación afectiva y la compañía, operando en gran medida como factor protector frente a un vínculo nocivo con la madre. Al interpretar este caso Lara-Quinteros señala que "el padre se encuentra ausente de los espacios cotidianos, pero presente en los espacios emocionales significativos de su hijo". 
Lo anterior es de gran relevancia para la identificación de lo que llamaremos el padre emocional, entendido como síntesis cultural del cambio desde un modelo de paternidad tradicional y hegemónica, representada socialmente por el rol del proveedor económico y la autoridad de la familia, hacia prácticas y sentidos que valoran la afectividad, la participación en el cuidado y la comunicación con los hijos/as. Como evidencia adicional de la emergencia del padre emocional podemos agregar los siguientes dichos de Lara-Quinteros: En otros relatos se visualiza cómo la figura del padre asume un rol de apoyo, o al menos de contención que contrasta con la postura materna... de alguna u otra forma el padre emerge en una lógica compensatoria que en varios trayectos biográficos permitió contener el desborde de la relación con la madre, lo que para los investigadores abre intersticios para resignificar aspectos de la ligazón padre-hijo y de la figura paterna por sí sola que antes se encontraban más solapados.

Finalmente Cosse señala que hubo, como era esperable, una reacción conservadora frente al nuevo modelo de paternidad expresada en organizaciones como La Liga de Madres y Padres de Familia, que promovían el retorno a los valores tradicionales de la paternidad, centralmente la autoridad patriarcal.

Algo parecido a este conservadurismo se asoma en los insultos y estigmatización que sufren los varones entrevistados por Lara-Quinteros y Avendaño, quienes en este sentido destacan los prejuicios que sus entrevistados enfrentan en tanto hijos frente a sus propios padres y madres y señalan que esto conduce a vivir no como un sujeto humano muchas veces sino como una especie de fantasma. Tal es el peso del estigma.

Para estos entrevistados es solo mediante el activismo político que se recupera la capacidad de vivir como cuerpos "reales" con iguales derechos. Existe esta misma convicción entre algunos de los padres mexicanos entrevistados por Laguna. Desde ahí el autor señala que se debe pensar el derecho al reconocimiento como una lucha continua, no eventualmente solo para un "nosotros".

Lo anterior nos lleva al tema de la acción política y nos permite pensar en las dinámicas de poder presentes en la construcción del cuerpo reproductivo, donde a veces la dominación masculina acierta el golpe respecto de los derechos de los hombres y paradójicamente privilegia las mujeres. Esto, por ejemplo, ocurre en el contexto de la sociedad argentina donde está prohibido para los varones el alquiler de vientre pero sí es legal para las mujeres.

Esta paradoja nos lleva a pensar en la necesidad de mirar cuál ha sido la historia propia de los hombres en las transformaciones de las masculinidades que hemos estado discutiendo. Así como el feminismo, en tanto artefacto de reflexión crítica vis a vis el movimiento de mujeres visibiliza como sujetos a las mujeres, la reflexión acerca de las masculinidades está llamada a visibilizar a determinados varones como impulsores del cambio en las relaciones de género desde el interior de procesos políticos autónomos, solidarios con el feminismo pero independientes. Tal es el caso, por ejemplo, de los hombres del movimiento homosexual.

\section{LA ACCIÓN DE LOS HOMBRES COMO FACTOR DEL CAMBIO EN LAS MASCULINIDADES}

A menudo se ha planteado la necesidad de ubicar la reciente transformación de la masculinidad en el marco de los cambios que ha generado el movimiento de mujeres hacia una mayor equidad en las relaciones de género. Pero hay quienes plantean (entre ellos Oscar Laguna, uno de los autores que aquí comentamos) que la oposición al patriarcado y su impacto en la 
construcción social de la masculinidad ha provenido también desde la lucha social y política del mundo homosexual, que por cierto incluye a muchos varones (Laguna Maqueda, 2013).

La transformación en los comportamientos y sentidos de la paternidad está determinada por ambas luchas, tanto la feminista como homosexual (más ampliamente el movimiento LGTB) y sin duda por otras circunstancias que aquí no viene al caso detallar.

Lo que aquí se pretende destacar es el papel protagónico que los propios hombres han tenido en las trasformaciones ocurridas dentro de los patrones socioculturales de lo masculino durante la segunda mitad del siglo XX y los comienzos del siglo XXI. De aquí la relevancia de la investigación concerniente a padres gays de Oscar Laguna y otras.

Laguna señala que la sociedad ha creado diversos mecanismos para mantener el orden. Específicamente agrega: para los aspectos relacionados a la sexualidad se han configurado diversos dispositivos ${ }^{3}$ y tecnologías de poder ${ }^{4}$, una de los cuales es la heteronormatividad, entendida esta última como una serie de reglas de comportamiento sexual y social, definidas y establecidas por la sociedad para regular los comportamientos y vínculos sexuales permitidos y avalados socialmente entre las personas, tanto del mismo sexo como de sexo distinto. A partir de dichos conceptos podemos entender el cuerpo reproductivo como parte de los mecanismos de control de la hetereonormatividad.

Según Laguna los varones gay trastocan los patrones heteronormativos y desestabilizan algunos de los patrones vinculados a la familia y a la crianza y cuidado al establecer relaciones filiales. Los varones gay aunque sufren de la negativa social en relación con la paternidad, desean desarrollar arreglos parentales y prácticas de cuidado muy similares a las familias que conocen y en las que fueron criados, no obstante la homofobia los impulsa a desapegarse de los modelos tradicionales de familia, con lo que de manera inadvertida desestabilizan algunas de las concepciones vinculadas a esta última.

Algunos de los prejuicios de los que habla Laguna, como por ejemplo que los varones no son capaces de procrear, criar ni educar niños/as, los podemos entender como mecanismos de naturalización de la masculinidad hegemónica que a su vez, inscritos dentro de una representación heteronormativa de la masculinidad, convierten la paternidad homosexual en una amenaza literalmente de "fin de mundo", de fin de procreación de la especie. Solo hay espacio para un imaginario del cuerpo masculino apto para la producción de las mercancías y la violencia pero sin "futuro" para la producción de la vida.

Como señala el autor, La homofobia ha creado al homosexual como un sujeto abyecto, un varón estéril que no desea reproducirse y no es capaz de criar infantes Debido a que se ha considerado a los homosexuales como sujetos estériles, por lógica se estima que es imposible que conformen una familia. Laguna agrega que estos fenómenos sociales, así como las prácticas homofóbicas, aunados a que no existen modelos de parentalidad gay, han hecho que muchos varones gay no consideren ni la crianza y cuidado de infantes ni la configuración

3 En el sentido foucaultiano como la red que se establece entre elementos de orden institucional, discursivo, moral, científico, filosófico, reglamentos, leyes y otros a través del cual se mantiene un orden social determinado.

4 Entendidas estas como aquellas tecnologías que determinan la conducta de los individuos, los someten a cierto tipo de fines o de dominación, y consisten en una objetivación del sujeto. 
de un arreglo parental como una vía posible de desarrollar su vida: nosotros estamos incluso más prejuiciados que los propios heterosexuales.

Señala Laguna: Los varones gay que acceden a los hijos lo hacen en los intersticios de los imperativos biológicos, las restricciones sociales y el imaginario social construido en torno a la homosexualidad.

Además, los padres gay mexicanos buscan "proteger"a sus hijos por medio de "burbujas" y "fachadas" que mantienen en secreto la naturaleza de la familia, manteniendo alejado los prejuicios y perjuicios que implica un medio homofóbico. Algo similar encontró Herrera en su investigación pionera sobre madres lesbianas en Santiago de Chile, quienes mantenían en secreto su relación hasta de los propios hijos/as para protegerlos de los perjuicios que podría provocarles una relación abiertamente lésbica. La implicancia de esta estrategia de "clandestinidad" es al menos problemática.

\section{EL CUERPO REPRODUCTIVO Y MÁS ALLÁ DEL GÉNERO}

El proceso de reproducción no solo produce seres humanos, también ayuda a reproducir el binarismo del sistema sexo/género. Una pregunta interesante radica en pensar las formas con que se coluden la heterosexualidad y el patriarcalismo para determinar cuáles son las expectativas sociales acerca del cuerpo masculino y la reproducción.

Completando el círculo volvamos a Bourdieu, quien señala que por estrecha que sea la correspondencia entre las realidades o los procesos del mundo natural y los principios de visión y de división que se les aplican, siempre queda lugar para una lucha cognitiva a propósito del sentido de las cosas del mundo y en especial de las realidades sexuales (Bourdieu, 2000).

En efecto, en oposición al orden de dominación masculina actualmente se manifiestan procesos globales de transformación de las identidades de género y de las prácticas sociales del parentesco que reflejan dicha lucha cognitiva y que por cierto se expresan en nuestra propia sociedad como en otras sociedades latinoamericanas.

Entre estos procesos son particularmente elocuentes las experiencia de vida de las personas transgénero. La reflexión en torno a la identidad de género trans como la parentalidad trans nos permite ver con mucha claridad cómo funcionan los mecanismos de naturalización en torno a la sexualidad, la identidad de género, la procreación y reproducción.

En este sentido Ilyssa Silfen (2014), en su trabajo The Reproductive Body: Exploring reproduction beyond gender, plantea que las personas trans que eligen ocupar sus órganos reproductivos biológicos para la reproducción nos obligan a cuestionar la autenticidad de los roles "naturales" de género, además de lo "natural" que pueda ser el binarismo sexo/ género, al comprobar que uno no necesariamente debe ser una mujer para dar a luz o que se deba ser hombre para convertirse en padre. Para Silfen la reproducción trans es un acto de rebeldía total frente al binarismo de sexo/género.

Al referirse al caso de Thomas Beatie, el primer hombre embarazado en Estados Unidos, Silfen señala que la figura del hombre embarazado hace visible la manera problemática en que caracterizamos a las personas de cuerpo/macho en tanto quien puede engendrar una prole y a la persona cuerpo/hembra como aquella que puede dar a luz, e incluso la manera 
más problemática aun en que hemos convertido a estas caracterizaciones en un aspecto esencial de nuestra interpretación de los cuerpos como macho o hembra.

Situada en un punto de crítica radical al binarismo sexo/género, el objetivo de la deconstrucción en Silfen es poder sacar al género de la reproducción, ya que la inhabilidad de reproducirse en tanto poseedor de un género que uno mismo ha definido, como es el caso de los cuerpos trans, es usado como significante de inautenticidad de género.

\section{BIBLIOGRAFÍA}

Alméras, D. (1997): Procesos de cambio en la visión masculina de las responsabilidades familiares. Séptima Conferencia Regional sobre la Integración de la Mujer en el Desarrollo Económico y Social de América Latina y el Caribe. Santiago CEPAL

Baker, G. (2008): La participación del hombre como padre en la región de Latinoamerica y el Caribe: una revisón de literatura crítica con consideraciones para políticas. Brasil : Promundo.

Bourdieu, P. (2000): La dominación masculina. Barcelona: ANAGRAMA.

Butler, J. (2002): Cuerpos que importan: Sobre los límites materiales discursivos del "sexo". Buenos Aires: Paidós.

Butler, J. (2006): Deshacer el género. Barelona: Paidós.

Cosse, I. (2009): La emergencia de un nuevo modelo de paternidad en Argentina (1950-1975). Estudios Demográficos y Urbanos, vol. 24, núm. 2, mayo-agosto, El Colegio de México, pp. 429-462.

Cultura Salud/eme (2011): Encusta IMAGES Chile. Santiago: Cultura salud/eme.

Garzón, J. S. (2012): Historia del Feminismo. Madrid: Catarata.

Haraway, D. (1988): Situated Knowledges: The Science Question in Feminism and the Privilege of. Feminist Studies, Vol. 14, No 3. pp. 575-599.

Harding, S. (1987): Is there a a Feminist Method? Bloomington/Indianapolis: Indiana University Press.

Herrera, F. (2015): Los hombres siempre adoptan: infertilidad y reproducción desde la perspectiva masculina. Actas del V Coloquio Internacional de Estudios sobre Varones y Masculinidades, (pág. S/N). Santiago de Chile.

Hochschild, A. (1989): The second Shift: Working Families and the revolution at home. New York: Penguin Books.

Laguna Maqueda, O. E. (2013): Vivir Contarcorriente. Arreglos parentales de varones gay en la ciudad de México. México: Librero de la administración pública.

Laguna Maqueda, O.E. (2015): Arreglos parentales de varones gay en la ciudad de México: entre la paternidad negada y la transformación imprevista. Actas del V Coloquio Internacional de Estudios sobre Varones y Masculinidades (pág. S/N). Santiago de Chile. 
Lara-Quinteros, R., \& Avendaño, M. F. (2015): Vínculo padre-hijo: un análisis desde el itinerario biográfico de varones jóvenes no heterosexuales. Actas del V Coloquio Internacional de Estudios sobre Varones y Masculinidades (pág. S/N). Santiago de Chile.

Libson, M. (2010): Parentalidades gays y lesbianas: una mirada sobre la discriminación y la exclusión. Cuadernos del Inadi, No 2 Agosto S/N p.p.

Robaldo, M. (2011): La homoparentalidad en la deconstrucción y reconstrucción de familia. Aportes para la discusión. Revista Punto Género, № 1, pp. 171-183.

Sandoval, M. L. (2002): Pierre Bourdieu y la teoría sobre la dominación masculina. Revista Colombiana de Sociología, Vol. VII, No 1, pp. 55-73.

Silfen, I. (2014): The reproductive body: Exploring reproducction beyond gender. City University of New York: Disertación y Tesis de Magister.

Valdés, T., \& Olavarría, J. (1998): Masculinidades y equidad de género en América Latina. Santiago de Chile: Flacso-Chile.

Wainerman, C. (2007): Conyugalidad y paternidad ¿Una revolución estancada?. En M. A. Gutiérrez, Género, familias y trabajo: rupturas y continuidades. Desafíos para la investigación política (págs. p.p. 179-222). Buenos Aires: CLACSO. 